\title{
Melatonin: a hepatoprotective agent against radioiodine toxicity in rats
}

\author{
Barlas $\mathrm{AM}^{1}$, Sadic $\mathrm{M}^{2}$, Atilgan $\mathrm{HI}^{2}$, Bag $\mathrm{YM}^{1}$, Onalan $\mathrm{AK}^{3}$, Yumusak $\mathrm{N}^{4}$, Senes $\mathrm{M}^{5}$, \\ Fidanci $\mathrm{V}^{5}$, Pekcici MR ${ }^{1}$, Korkmaz $\mathrm{M}^{2}$, Kismet $\mathrm{K}^{1}$, Koca $\mathrm{G}^{2}$ \\ Ankara Education and Research Hospital, Department of General Surgery, Ankara, Turkey. \\ kemalkismet@yahoo.com
}

\section{ABSTRACT}

OBJECTIVES: The aim of the current study was to investigate the possible radioprotective effects of melatonin against hepatic radioiodine (RAI) toxicity.

METHODS: Thirty-six rats were randomly divided into three groups: untreated control (Group 1); oral radioiodine (RAl, $111 \mathrm{MBq}$ ) administrated rats (Group 2), and melatonin group (oral RAl and daily intraperitoneal injection of $12 \mathrm{mg} / \mathrm{kg}$ melatonin-Group 3). In the third group, melatonin administration was started two days before and continued for five days after RAI administration. Twenty-four hours after the administration of the last dose of melatonin, liver samples were taken for biochemical and histopathological evaluation.

RESULTS: Oxidative stress parameters demonstrated that melatonin treatment decreased the tissue malondialdehyde (MDA), advanced the oxidation protein products (AOPP) levels, and increased the total-SH (sulphydryl) levels when compared with RAI group. The differences were statistically significant between these groups for all parameters $(p<0.05)$. The histopathological damage in the melatonin-treated group was significantly less than the damage in RAl group ( $p<0.05$ for all pathological parameters).

CONCLUSION: The results of this study demonstrated that melatonin reduced the harmful effects of RAI treatment on the liver. Anti-inflammatory and antioxidant activities are likely to be involved in the mechanism underlying the radio-protective effects of melatonin (Tab. 3, Fig. 1, Ref. 30). Text in PDF www.elis.sk.

KEY WORDS: melatonin, radioiodine, liver, oxidative stress, histopathology.

\section{Introduction}

Iodine-131 (I-131) is a well-known and widely used radionuclide which radiates beta as well as gamma rays. High dose I-131 targeted radionuclide therapy is associated with hematological toxicity. There are also known to be non-hematological toxicities from the use of I-131 therapy including transient nausea and vomiting, sialoadenitis, transient hepatic abnormalities, lacrimal gland dysfunction, and hypothyroidism of differing degrees. Thyroid and liver functions are of specific importance after I-131 administration because the radioactivity is generally concentrated in these organs via sodium/iodide symporter (NIS) system. However, there is limited information available to be able to evaluate the acute and late effects of I-131 therapy on thyroid and liver functions (1). Cell death caused by the ionizing radiation is achieved through free radical formation. In patients with thyroid cancer, it is known

${ }^{1}$ Ankara Education and Research Hospital, Department of General Surgery, Ankara, Turkey, ${ }^{2}$ Ankara Education and Research Hospital, Department of Nuclear Medicine, Ankara, Turkey, ${ }^{3}$ Siirt State Hospital, Department of General Surgery, Siirt, Turkey, ${ }^{4}$ Harran University, Faculty of Veterinary Medicine, Department of Pathology, Sanliurfa, Turkey, and ${ }^{5}$ Ankara Education and Research Hospital, Department of Biochemistry, Ankara, Turkey

Address for correspondence: K. Kismet, MD, Ankara Egitim ve Arastirma Hastanesi Genel Cerrahi Klinigi, Ulucanlar; Ankara.

Phone: +90.312.5953885, Fax: +90.312.3633330 that oral radioiodine (RAI) therapy following thyroidectomy can induce oxidative stress, resulting in substantial damage to cellular components such as lipids, proteins and DNA (2).

Melatonin (N-acetyl-5-methoxytryptamine) is a powerful antioxidant produced by the pineal gland and various other organs including the ovaries, testes, bone marrow, gut, placenta, and liver. Although melatonin was first considered to solely be a circadian cycle regulator, several studies have shown that it is effective as a free radical scavenger and antioxidant agent. In addition to the regulation of circadian biology, melatonin has also been shown to modulate several molecular pathways of inflammation, oxidative stress, and cellular injury and have vasorelaxant and anti-fibrotic properties $(3,4)$.

Various studies have demonstrated the hepatoprotective effects of melatonin against numerous conditions, including hepatic steatosis (5), hepatic ischemia/reperfusion injury (6), fructosemediated metabolic syndrome model (7), exposure to microwave radiation (8), non-alcoholic fatty liver disease (9), carbon tetrachloride-induced liver fibrosis (10), and thioacetamide-induced liver fibrosis (11).

With the consideration of antioxidant and anti-inflammatory properties of melatonin, the aim of the current study was to investigate the possible radioprotective effects of melatonin against hepatic I-131 toxicity. To the best of our knowledge, this is the first study to evaluate the protective effect of melatonin against 
the damage and oxidative stress generating effects of I-131 on liver tissue.

\section{Materials and methods}

This study was conducted in the Husnu Sakal Experimental and Clinical Practice Center, Ankara, Turkey. All procedures were performed in accordance with the National Guidelines for the Use and Care of Laboratory Animals. Approval for the study was granted by the Local Animal Ethics Committee of Ankara Training and Research Hospital (Ankara, Turkey).

\section{Animals}

The study included 36 female Wistar-Albino rats, each weighing $250 \pm 25 \mathrm{~g}$. Before starting the study, the rats had one week of adaptation to laboratory conditions. The animals were housed under standard laboratory conditions at a constant temperature of $21 \pm$ $2{ }^{\circ} \mathrm{C}$ and a 12 -hour light/dark cycle. All the rats were kept in polypropylene cages with disposable absorbent cloths under sterile paddy husks to avoid contamination from radioactive urine. The animals were fed with standard laboratory chow and water ad libitum.

The study entitled 'Hepatoprotective effect of dexmedetomidine against radioiodine toxicity in rats: Evaluation of oxidative status and histopathological changes' (12) published by the same research team was conducted at the same time and under the same conditions in accordance with the Experimental Animal Research Regulations. The biochemical and histopathological results of Group 1 (untreated control) and Group 2 (radioiodine) were used by both that study and the current study.

\section{Experimental design}

The rats were randomly divided into 3 groups of 12 . The first group was the untreated control (UC) group, which received no RAI therapy or melatonin $(\mathrm{n}=12)$. Radioiodine (Mon-Iyot-131 Eczacıbaşı/Monrol Nukleer Urunler Sanayi ve Ticaret Anonim Sirketi Gebze, Kocaeli, Turkey) was applied at $111 \mathrm{MBq}$ (3 mCi) after replacing the orogastric catheter in the rats in Groups 2 and 3. No other medication was given to the rats in Group 2 (RAI group, $\mathrm{n}=12$ ). The rats in the Group 3 (melatonin group, $\mathrm{n}=12$ ) were treated with a daily intraperitoneal injection of $12 \mathrm{mg} / \mathrm{kg}$ body weight melatonin (Melatonina ${ }^{\circledR}$, Przedsiebiorstwo Farmaceutyczne, Zakroczym, Poland) which was started 2 days before the RAI administration and was continued for 5 days after RAI. At hour 24 after the administration of the last dose of melatonin, the animals were sacrificed by high dose of anesthetic. Liver samples were taken for biochemical and histopathological evaluation.

\section{Evaluation of oxidative stress}

The evaluation of oxidative stress parameters was performed in the Biochemistry Department of Ankara Training and Research Hospital. Tissues were stored at $-80^{\circ} \mathrm{C}$ until assays. Measurements were taken of tissue malondialdehyde (MDA), total-SH (sulphydryl), and advanced oxidation protein products (AOPP) levels.

Rat liver tissues were weighted and homogenized in 10 volumes of cold phosphate buffer saline (PBS; $50 \mathrm{mM}$, pH 7.4) using an automatic homogenizer (Heidolph DIAX 900). MDA levels were calculated using the fluorometric method as described by Wasowicz et al (13). Following the thiobarbituric acid (TBA) reaction with MDA, the reaction product was extracted in butanol and measured spectrofluorometrically at wavelengths of $525 \mathrm{~nm}$ for excitation and $547 \mathrm{~nm}$ for emission. A standard $0-5 \mu \mathrm{mol} / \mathrm{L}$ 1,1,3.3-tetraethoxypropane solution was used. For the measurement of tissue MDA levels, $50 \mu \mathrm{L}$ of homogenate was added and introduced into $10 \mathrm{~mL}$ glass tubes containing $1 \mathrm{~mL}$ of distilled water. After the addition of $1 \mathrm{~mL}$ of the solution containing 29 $\mathrm{mmol} / \mathrm{L}$ TBA in acetic acid and mixing, the samples were placed in a water bath and heated for $1 \mathrm{~h}$ at $95-100^{\circ} \mathrm{C}$. The samples were then cooled, $25 \mu \mathrm{L}$ of $5 \mathrm{~mol} / \mathrm{L}$ hydrochloric acid (HCL) was added and the reaction mixture was extracted by agitation for $5 \mathrm{~min}$ with $3.5 \mathrm{~mL}$ n-butanol. After separation of the butanol phase by centrifugation at $1500 \mathrm{x}$ g for $10 \mathrm{~min}$, the fluorescence of the butanol extract was measured with a fluorometer (HITACHI F-2500) at wave-lengths of $525 \mathrm{~nm}$ for excitation and $547 \mathrm{~nm}$ for emission. 0-5 $\mu \mathrm{mol} / \mathrm{L} 1.1$ ',3.3'-tetraethoxypropane solutions were used as standard. MDA levels were presented as $\mu \mathrm{mol} / \mathrm{g}$ wet tissue (13).

Total SH groups were measured spectrophotometrically using the Sedlak and Lindsay method (14). Aliquots of $250 \mu 1$ of the supernatant fraction of the tissue homogenate were mixed in $5 \mathrm{~mL}$ test tubes with $750 \mu \mathrm{l}$ of $0.2 \mathrm{M}$ Tris buffer, $\mathrm{pH} 8.2$, and $50 \mu \mathrm{l}$ of $0.01 \mathrm{M} 5,5$ "-dithiobis (2-nitrobenzoic acid) (DTNB). With the addition of $3950 \mu \mathrm{l}$ of absolute methanol. The mixture was brought to $5 \mathrm{~mL}$. A reagent blank (without sample) and a sample blank (without DTNB) were prepared in a similar manner. The test tubes were stoppered with rubber caps, the color was let to develop for 15 min and the reaction mixtures were centrifuged at approximately $3,000 \mathrm{~g}$ at room temperature for $15 \mathrm{~min}$. The absorbance of supernatant fractions was read in a spectrophotometer at $412 \mathrm{~nm}$ (14).

AOPP were determined according to method described by Witko-Sarsat et al (15). AOPP were measured by spectrophotometrically and were calibrated with Cloramin-T solutions that in the presence of potassium iodide absorb at $340 \mathrm{~nm}$. In standard tubes, $50 \mu \mathrm{L}$ of $1.16 \mathrm{M}$ potassium iodide (KI) was added to 1000 $\mu \mathrm{L}$ of chloramine-T solution ( $0-100 \mathrm{mmol} / \mathrm{liter})$ followed by 100 $\mu \mathrm{L}$ of acetic acid. In test tube, $1000 \mu \mathrm{L}$ of tissue homogenate diluted $1 / 5$ in PBS, $50 \mu \mathrm{L} 1.16 \mathrm{M} \mathrm{KI}$ and $100 \mu \mathrm{L}$ of acetic acid were mixed and the absorbance of the reaction mixture is immediately read at $340 \mathrm{~nm}$ on the spectrophotometer against blank containing $1000 \mu \mathrm{L}$ PBS, $100 \mu \mathrm{L}$ acetic acid and $50 \mu \mathrm{L} 1.16 \mathrm{M} \mathrm{KI}$.

\section{Histopathological examination}

The histopathological analyses were performed at the Pathology Department of Harran University Faculty of Veterinary Medicine. For light microscopic analyses, the samples were obtained from the liver and fixed in $10 \%$ neutral buffered formalin solution for 2 days. The tissues were washed in running water and were dehydrated with increasing concentrations of ethanol $(50 \%, 75$ $\%, 96 \%, 100 \%)$. After dehydration, the specimens were put into xylene to obtain transparency and were then infiltrated with and embedded in paraffin. The embedded tissues were cut into $5-\mu \mathrm{m}$ thick sections using a Leica RM 2125 RT microtome and stained 
Tab. 1. Grading of histopathological changes of the groups.

\begin{tabular}{|c|c|c|}
\hline $\begin{array}{l}\text { Hyperemia } \\
\text { Grade 0: no hyperemia } \\
\text { Grade 1: mild hyperemia } \\
\text { Grade 2: severe hyperemia } \\
\text { Grade 3: very severe hyperemia }\end{array}$ & $\begin{array}{l}\text { Presence of inflammatory cells } \\
\text { Grade } 0 \text { : absent } \\
\text { Grade 1: few inflammatory cells } \\
\text { Grade 2: severe inflammatory cells } \\
\text { Grade 3: very severe inflammatory cells }\end{array}$ & $\begin{array}{l}\text { Steatosis (Microvesicular) } \\
\text { Grade 0: absent } \\
\text { Grade 1: mild steatosis } \\
\text { Grade 2: severe steatosis } \\
\text { Grade 3: very severe steatosis }\end{array}$ \\
\hline $\begin{array}{l}\text { Cellular Changes (Multiple Nucleus) } \\
\text { Grade 0: absent } \\
\text { Grade 1: mild cellular changes } \\
\text { Grade 2: severe cellular changes } \\
\text { Grade 3: very severe cellular changes }\end{array}$ & $\begin{array}{l}\text { Bile Duct Proliferation } \\
\text { Grade 0: absent } \\
\text { Grade 1: mild proliferation } \\
\text { Grade 2: severe proliferation } \\
\text { Grade 3: very severe proliferation }\end{array}$ & $\begin{array}{l}\text { Fibrosis } \\
\text { Grade 0: absent } \\
\text { Grade 1: mild fibrosis } \\
\text { Grade 2: severe fibrosis } \\
\text { Grade 3: very severe fibrosis }\end{array}$ \\
\hline $\begin{array}{l}\text { Venous Lesions } \\
\text { Grade 0: absent } \\
\text { Grade 1: mild lesions } \\
\text { Grade 2: severe lesions } \\
\text { Grade 3: very severe lesions }\end{array}$ & $\begin{array}{l}\text { Capsule thickening } \\
\text { Grade 0: absent } \\
\text { Grade 1: mild thickening } \\
\text { Grade 2: severe thickening } \\
\text { Grade 3: very severe thickening }\end{array}$ & $\begin{array}{l}\text { Granuloma formation } \\
\text { Grade 0: absent } \\
\text { Grade 1: mild granuloma formation } \\
\text { Grade 2: severe granuloma formation } \\
\text { Grade 3: very severe granuloma formation }\end{array}$ \\
\hline
\end{tabular}

with hematoxylin\&eosin. Histopathological examinations were performed with a light microscope (Olympus BX-50, Tokyo, Japan) by a pathologist blinded to the study design. Histopathological parameters including hyperemia, presence of inflammatory cells, steatosis, capsule thickening, cellular changes (multiple nucleus), bile duct proliferation, fibrosis, venous lesions (endothelial loss, subintimal thickening and fibrosis), and granuloma formation were evaluated semiquantitatively according to modified histological activity index (HAI) $(16,17)$. The scoring system of these parameters are given in Table 1.

\section{Statistical analysis}

Data analysis was performed using the Statistical Package for Social Sciences (SPSS) version 15.0 for Windows (SPSS Inc, Chicago, IL). All variables were normally distributed about the mean. Data were presented as mean $\pm \mathrm{SD}$. Differences between the groups were evaluated by one-way analysis of variance (ANOVA) or Kruskal-Wallis variance analysis, whichever was appropriate. When the $p$ values from the variance analysis were statistically significant, the Tukey honestly significant difference (HSD) or Mann-Whitney U multiple comparison test was used to determine which group was different from the others. A value of $p<0.05$ was considered to be statistically significant.

\section{Results}

\section{Oxidative stress parameters}

The mean levels of the oxidative stress parameters of the liver (MDA, total SH, and AOPP) are summarized in Table 2.

There was a significant difference in tissue MDA levels between the RAI group and the other groups $(\mathrm{p}<0.05)$. The highest tissue MDA levels were detected in the RAI group. The levels in the melatonin group were significantly lower than those of the RAI group $(p=0.012)$. The MDA levels were lower in the untreated control group than in the melatonin group, but the difference was not statistically significant $(\mathrm{p}>0.05)$.

Just as in the MDA level comparison, total tissue SH levels were significantly different between the RAI group and the other
Tab. 2. Mean oxidative stress parameter levels of the groups.

\begin{tabular}{lccc}
\hline GROUPS & MDA & TOTAL SH & AOPP \\
\hline Group 1 (Control) & $2.28 \pm 0.24$ & $157.37 \pm 22.90$ & $15.50 \pm 3.14$ \\
Group 2 (RAI) & $3.16 \pm 0.47^{\mathrm{a}}$ & $114.29 \pm 15.28^{\mathrm{a}}$ & $29.20 \pm 2.45^{\mathrm{a}}$ \\
Group 3 (Melatonin) & $2.43 \pm 0.66^{\mathrm{b}}$ & $149.42 \pm 23.94^{\mathrm{b}}$ & $23.32 \pm 4.41^{\mathrm{a}, \mathrm{b}}$ \\
\hline
\end{tabular}

a - Significantly different, control versus other groups; ${ }^{b}$ - Significantly different, RAI versus melatonin group

groups $(\mathrm{p}<0.05)$. Total tissue SH levels were lowest in the RAI group. The levels of the melatonin group were higher than those of the RAI group and the difference was statistically significant $(p=0.001)$. Although the total SH levels were higher in the untreated control group when compared with the melatonin group, no significant difference was found between these groups $(\mathrm{p}>$ $0.05)$.

There was a significant difference in tissue AOPP levels between the RAI group and the other groups $(p<0.05)$. The highest tissue AOPP levels were determined in the RAI group. The levels of the melatonin group were significantly lower than those of the RAI group $(p=0.001)$. The AOPP levels were lower in the untreated control group than those of the melatonin group and the difference was statistically significant $(\mathrm{p}=0.001)$.

\section{Histopathological results}

The mean scores of the histological activity index (HAI) of the groups are given in Table 3. Statistical analyses of the HAI scores demonstrated that the histopathological damage in the melatonin-treated group was significantly smaller than the that in the RAI group ( $p<0.05$ for all pathological parameters). The HAI scores of the untreated control group were significantly lower than those of the RAI group ( $p<0.05$ for all parameters). There was a statistically significant difference between the untreated control and melatonin groups only in respect of the inflammation score, which was significantly lower in the untreated control group than in the melatonin group $(\mathrm{p}=0.039)$. No difference was determined between the untreated control and melatonin groups in respect of the mean scores of the other pathological parameters $(\mathrm{p}>0.05)$ (Tab. 3). 
Tab. 3. Mean pathological scores of the groups.

\begin{tabular}{lccc}
\hline & $\begin{array}{c}\text { Group 1 } \\
\text { (Control) }\end{array}$ & $\begin{array}{c}\text { Group 2 } \\
\text { (RAI) }\end{array}$ & $\begin{array}{c}\text { Group 3 } \\
\text { (Melatonin) }\end{array}$ \\
\hline Hyperemia & $0.08 \pm 0.02$ & $1.41 \pm 0.41^{\mathrm{a}}$ & $0.50 \pm 0.18^{\mathrm{b}}$ \\
Inflammation & $0.00 \pm 0.00$ & $1.33 \pm 0.38^{\mathrm{a}}$ & $0.50 \pm 0.18^{\mathrm{a}, \mathrm{b}}$ \\
Steatosis & $0.08 \pm 0.02$ & $1.41 \pm 0.41^{\mathrm{a}}$ & $0.41 \pm 0.07^{\mathrm{b}}$ \\
Capsular thickening & $0.00 \pm 0.00$ & $1.16 \pm 0.24^{\mathrm{a}}$ & $0.25 \pm 0.12^{\mathrm{b}}$ \\
Cellular changes & $0.00 \pm 0.00$ & $1.08 \pm 0.21^{\mathrm{a}}$ & $0.33 \pm 0.17^{\mathrm{b}}$ \\
Bile duct proliferation & $0.00 \pm 0.00$ & $0.91 \pm 0.18^{\mathrm{a}}$ & $0.00 \pm 0.00^{\mathrm{b}}$ \\
Fibrosis & $0.00 \pm 0.00$ & $1.33 \pm 0.38^{\mathrm{a}}$ & $0.16 \pm 0.05^{\mathrm{b}}$ \\
Venous lesions & $0.00 \pm 0.00$ & $1.08 \pm 0.21^{\mathrm{a}}$ & $0.08 \pm 0.02^{\mathrm{b}}$ \\
Granuloma formation & $0.00 \pm 0.00$ & $1.41 \pm 0.41^{\mathrm{a}}$ & $0.16 \pm 0.05^{\mathrm{b}}$ \\
\hline
\end{tabular}

a - Significantly different, control versus other groups; ${ }^{\mathrm{b}}$ - Significantly different, RAI versus melatonin group
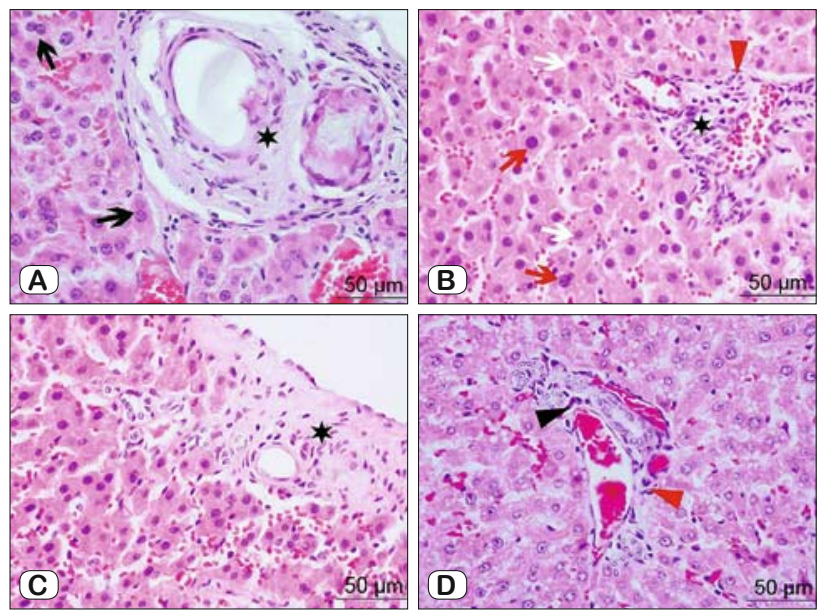

Fig. 1. Histopathological findings of the RAI and melatonin groups. A - RAI group: Capsulated granuloma formation (star) and dense double-nucleated hepatocytes (arrows) in the rat liver tissue. H\&E. X400; B - RAI group: Severe perivascular and interstitial inflammatory cell infiltration (star) with severe fibrosis (red arrow-head), and many hepatocytes with macronucleus (red arrow) and micronucleus structure (white arrow), H\&E, X400; C - Melatonin group: Underdeveloped granuloma formation (star) in the rat liver tissue, $H \& E$, X400; D - Melatonin group: Mild perivascular inflammation (black arrow-head) and fibrosis (red arrow-head), H\&E, X400).

The histopathological findings are presented in the Figure. Liver tissue sections from the untreated control group exhibited almost normal morphology. RAI caused marked hyperemia, inflammatory cell infiltration, steatosis, capsule thickening, cellular changes, bile duct proliferation, fibrosis, venous lesions, and granuloma formation. It was observed that melatonin significantly diminished the histopathological abnormalities when compared with the RAI group.

\section{Discussion}

The recommended treatment for most patients diagnosed with papillary and follicular thyroid carcinoma is thyroidectomy followed by RAI ablation. The objectives of RAI treatment include the elimination of residual normal or tumoral thyroid cells, an improvement in the sensitivity of serum thyroglobulin assays, greater sensitivity of detection of locoregional and/or metastatic disease on follow-up RAI whole-body scans, and for post-treatment scans to identify additional previously undetected foci of disease. Although RAI therapy is generally well tolerated, complications may develop such as transient neck pain and edema, dysfunction in the pulmonary, gastrointestinal, and hematopoietic systems, salivary glands, nasolacrimal apparatus and gonads, in addition to second primary malignancies. These complications are rarely life-threatening, but they can have a negative effect on the quality of life of the patient (18).

Despite the general toxicity of radioactive I-131 to thyroid cells that accumulate iodine from the bloodstream, it can be biodistributed to other organs and the entire body. In patients who have undergone total thyroidectomy, the lower gastrointestinal tract, kidney, stomach, heart wall and liver have been seen to absorb relatively high doses per unit of administered iodine activity (19). It has been reported that post-therapy scans often reveal diffuse I-131 uptake in the liver $(18,20)$.

Few studies have evaluated the harmful effects and hepatotoxicity of I-131. In a study by Vasil'ev et al. (21) investigating the liver and kidney functions of thyroid cancer patients on RAI therapy, a decrease in absorptive and secretory hepatocytic function, and a decrease in total renal function after RAI therapy were determined on hepatography and renography. These changes were stated to be stable and at a moderate level.

In a study of rat livers, Atilgan et al (22) demonstrated that I-131 resulted in histopathological changes such as hyperemia, steatosis, fibrosis, and capsule thickening in the third month following I-131 treatment. Montelukast sodium was also found to be effective in protecting the liver against the morphological damage resulting from the administration of I-131.

Jhummon et al (23) reported two cases of extremely uncommon but severe complications of RAI treatment. Liver toxicity associated with I-131 developed in previously healthy patients with Grave's disease, and provision of hepatoprotective treatment regimens, recovery was obtained. It was concluded that although hepatic toxicity after RAI treatment was not a common event, physicians should be aware of this potential complication.

The formation of reactive oxygen species and reactive nitrogen species results from exposure of biological systems to ionizing radiation. These free radicals react with cellular macromolecules such as DNA, RNA, proteins, membrane etc., causing cell dysfunction and mortality. In addition to these reactions in the tumor, they can also occur in normal cells when exposed to radiation. Depending on the dose, exposure to high doses of ionizing radiation results in damage to the hematopoietic, gastrointestinal or central nervous systems. As the level of cell turnover in the hematopoietic system is high, it is one of the most radiosensitive tissues in the body. Compared to hematopoietic syndrome, higher doses of irradiation are required to induce gastrointestinal and central nervous syndromes $(24,25)$.

It is known that reactive oxygen species (ROS) are involved in a variety of cellular processes ranging from physiological to pathological responses. Not only cell survival, proliferation and differentiation are driven by ROS at the physiological level, but also cell death by apoptosis or necrosis at the higher level. An imbalance between cellular oxidant species and antioxidants is 
known as oxidative stress and this has a direct toxic effect on cells, leading to lipid peroxidation, protein oxidation or DNA damage. In almost all human pathologies, oxidative stress is seen to play a causative or adjuvant role, including cancer, neurodegeneration, aging, and chronic inflammatory pathologies (26).

In a study by Ran et al (27), it was demonstrated that wholebody irradiation resulted in a substantial increase in the MDA level, while there was a significant decline in antioxidant enzyme activity (SOD and CAT) and reduced antioxidant molecular levels (GSH) in the liver and spleen. The administration of Dragon's blood and the associated extracts prior to radiation were found to effectively mitigate oxidative stress in the liver and spleen.

Healthy cells and tissues can be protected from the harmful effects of radiation by prophylactic radio-protectors. However, the research to date on synthetic radio-protectors has brought little success, mainly due to various toxicity-related problems. Therefore, no medication has been yet widely accepted in clinical practice.

Melatonin (N-acetyl-5-methoxytryptamine), is the hormone of the pineal gland, with a circadian rhythm generated by the circadian pacemaker located in the suprachiasmatic nucleus (SCN) of the hypothalamus, and which is synchronized to 24 hours by the light-dark cycle acting via the SCN. Serum melatonin concentrations are low during the day and increase significantly at night. Melatonin synthesis is strictly controlled by lighting conditions. When synthesized, melatonin is not stored in pineal cells but is rapidly released into the bloodstream and then into other body fluids, such as bile, cerebrospinal fluid, saliva, semen, amniotic fluid and ovarian follicular fluid. The many functions of melatonin in organisms include helping to synchronize circadian rhythms, sleep promotion, immune stimulation, blood pressure regulation, seasonal reproductive regulation, oncostatic function, and antidepressive function (26).

Melatonin is a powerful free radical scavenger and a broadspectrum antioxidant. As it has small dimensions and highly lipophilic properties, melatonin is able to cross all cell membranes and easily reach subcellular compartments, including mitochondria and nuclei, where it seems to accumulate in high concentrations. Lipid peroxidation and damage to protein, and DNA are prevented by melatonin and it has been noted that optimal mitochondrial function and homeostasis are preserved by melatonin through the reduction and prevention of mitochondrial oxidative stress, with a subsequent curtailment of apoptotic events and cell death (26).

Melatonin also has significant anti-inflammatory and immunomodulatory effects and modulates the processes of angiogenesis and wound healing. The anti-inflammatory activity of melatonin depends on the inhibition of iNOS and mitochondrial iNOS expression. The activation of the pro-inflammatory enzymes COX-2 and iNOS in glioma cells is also specifically prevented by melatonin without simultaneous inhibition of COX-1 enzyme, thereby indicating an anti-inflammatory action (28). Using the livers of elderly female rats, Kireev et al. (29) researched the effects of aging and ovariectomy on various physiological parameters related to inflammation and oxidative stress and whether long-term administration of melatonin had any effect. Melatonin, administered to both intact and ovariectomized rats, was seen to significantly reduce the levels of $\mathrm{NO}_{x}, \mathrm{LPO}$ and pro-inflammatory cytokines in the liver compared to the untreated rats. A significant increase in IL-10 and reductions in iNOS, HO-1, IL-6, TNF- $\alpha$ and IL-1 $\beta$ protein expression were also determined in the rats treated with melatonin.

In respect of hepatoprotective effects, numerous articles have demonstrated the successful use of exogenous melatonin to treat many different pathophysiological conditions, including hepatic steatosis (5), hepatic ischemia/reperfusion injury (6), fructosemediated metabolic syndrome model (7), exposure to microwave radiation (8), non-alcoholic fatty liver disease (9), carbon tetrachloride-induced liver fibrosis (10), and thioacetamide-induced liver fibrosis (11). The hepatoprotective action of melatonin has been largely attributed to its antioxidant effects. Hepatic lipid peroxidation (LPO), as measured through malondialdehyde levels has been shown to be highly attenuated by melatonin. Furthermore, melatonin also seems to increase the activity and/or expression of hepatic antioxidant enzymes such as superoxide dismutase (SOD), glutathione (GSH), and glutathione peroxidase (GPx) after most types of injury and increase hepatic catalase. The increase in serum enzyme levels of aspartate transaminase, alanine transaminase, lactate dehydrogenase, alkaline phosphatase, $\gamma$ glutamyl transferase and bilirubin, indicating the extent of cell damage, has been seen to be reduced with the administration of melatonin. The results of histopathology examinations have demonstrated that animals treated with melatonin typically present with reduced hepatocellular necrosis or attenuated infiltration of polymorphonuclear granulocytes. Decreased hepatic levels of myeloperoxidase also suggest that neutrophil granulocyte infiltration is greatly reduced by melatonin. The hepatoprotective effects are exerted through suppression of the pro-inflammatory pathway of the immune response, in other words, through anti-inflammatory effects (30).

There is a wide range of opinion concerning both the route of melatonin administration, as well as the dose, the latter ranging a thousand-fold from $100 \mu \mathrm{g} / \mathrm{kg}$ to $100 \mathrm{mg} / \mathrm{kg}$. Very few data are available on dose-response relationships, and most studies have not included measurements of plasma melatonin levels (30). In the present study, melatonin was administered at the dose of 12 $\mathrm{mg} / \mathrm{kg} /$ day with daily intraperitoneal injections.

In the light of the known antioxidant, anti-inflammatory, and, hepatoprotective activities of melatonin, the hypothesis of the present study was that melatonin would be effective against liver injury induced by oxidative stress and inflammation after RAI treatment. Histopathological parameters including hyperemia, inflammation, steatosis, capsular thickening, cellular changes, bile duct proliferation, fibrosis, venous lesions, and granuloma formation, showed that melatonin protected the rat liver against RAI-related liver damage. The mean scores of all these histopathological parameters were lower in the melatonin-treated rats and there was a significant difference between the melatonin and RAI groups ( $p$ $<0.05)$. Oxidative stress parameters demonstrated that melatonin treatment decreased the MDA and AOPP levels, and increased the total SH levels when compared with RAI group. The results of the current study, in accordance with the results of previously conducted studies, indicated that melatonin had significant antiinflammatory and antioxidant activities. 
$95-100$

To the best of our knowledge, this is the first study to evaluate the radioprotective effects of melatonin on the liver. The results of this study demonstrated that melatonin reduced the harmful effects of RAI treatment on the liver. Anti-inflammatory and antioxidant activities are likely to be involved in the mechanism underlying the radioprotective effects of melatonin, which can be considered for use as a hepatoprotective agent against the adverse effects of RAI therapy.

\section{Learning points}

- Melatonin reduced the harmful effects of RAI treatment on the liver.

- Anti-inflammatory and antioxidant activities are likely to be involved in the mechanism underlying the radioprotective effects of melatonin.

- Melatonin can be considered for use as a hepatoprotective agent against the adverse effects of RAI therapy.

\section{References}

1. Quach A, Ji L, Mishra V et al. Thyroid and hepatic function after high-dose 131 I-metaiodobenzylguanidine (131I-MIBG) therapy for neuroblastoma. Pediatr Blood Cancer 2011; 56: 191-201.

2. Bhartiya US, Raut YS, Joseph LJ, Hawaldar RW, Rao BS. Evaluation of the radioprotective effect of turmeric extract and vitamin $\mathrm{E}$ in mice exposed to therapeutic dose of radioiodine. Indian J Clin Biochem 2008; 23: 382-386.

3. Esteban-Zubero E, Alatorre-Jiménez MA, López-Pingarrón L et al. Melatonin's role in preventing toxin-related and sepsis-mediated hepatic damage: A review. Pharmacol Res 2016; 105: 108-120.

4. Hu W, Ma Z, Jiang S et al. Melatonin: the dawning of a treatment for fibrosis? J Pineal Res 2016; 60: 121-131.

5. Sun H, Huang FF, Qu S. Melatonin: a potential intervention for hepatic steatosis. Lipids Health Dis 2015; 14: 75.

6. Deng WS, Xu Q, Liu YE, Jiang CH, Zhou H, Gu L. Effects of melatonin on liver function and lipid peroxidation in a rat model of hepatic ischemia/reperfusion injury. Exp Ther Med 2016; 11: 1955-1960.

7. Demirtas CY, Pasaoglu OT, Bircan FS, Kantar S, Turkozkan N. The investigation of melatonin effect on liver antioxidant and oxidant levels in fructose-mediated metabolic syndrome model. Eur Rev Med Pharmacol Sci 2015; 19: 1915-1921.

8. Djordjevic B, Sokolovic D, Kocic G et al. The effect of melatonin on the liver of rats exposed to microwave radiation. Bratisl Lek Listy 2015; 116: $96-100$.

9. Stacchiotti A, Favero G, Lavazza A et al. Hepatic macrosteatosis is partially converted to microsteatosis by melatonin supplementation in ob/ ob mice non-alcoholic fatty liver disease. PLoS One 2016; 11: e0148115.

10. Kang JW, Hong JM, Lee SM. Melatonin enhances mitophagy and mitochondrial biogenesis in rats with carbon tetrachloride-induced liver fibrosis. J Pineal Res 2016; 60: 383-393.

11. Czechowska G, Celinski K, Korolczuk A et al. Protective effects of melatonin against thioacetamide-induced liver fibrosis in rats. J Physiol Pharmacol 2015; 66: 567-579.
12. Kismet K, Sadic M, Bag YM et al. Hepatoprotective effect of dexmedetomidine against radioiodine toxicity in rats: Evaluation of oxidative status and histopathological changes. Int Surg. 2016 Mar 28. DOI: 10.9738/ INTSURG-D-15-00325.1.

13. Wasowicz W, Neve J, Peretz A. Optimized steps in fluorometric determination of thiobarbituric acid-reactive substance in serum: Importance of extraction $\mathrm{pH}$ and influence of sample preservation and storage. Clin Chem 1993; 39: 2522-2526.

14. Sedlak J, Lindsay RH. Estimation of total, protein bound, and nonprotein sulfhydryl groups in tissue with Ellman's reagent. Anal Biochem 1968; 25: 192-205.

15. Witko-Sarsat V, Friedlander M, Nguyen Khoa T et al. Advanced oxidation protein and products as novel mediators of inflammation and monocyte activation in chronic renal failure. J Immunol 1998; 161: 2524-2532.

16. Ishak K, Baptista A, Bianchi $L$ et al. Histological grading and staging of chronic hepatitis. J Hepatol 1995; 22: 696-699.

17. Ishak KG. Pathologic features of chronic hepatitis. A review and update. Am J Clin Pathol 2000; 113: 40-55.

18. Fard-Esfahani A, Emami-Ardekani A, Fallahi B et al. Adverse effects of radioactive iodine-131 treatment for differentiated thyroid carcinoma. Nucl Med Commun 2014; 35: 808-817.

19. Changlai SP, Chang PJ, Chen CY. Biodistribution and dosimetry of (131)I in thyroidectomy patients using semiquantitative gamma-camera imaging. Cancer Biother Radiopharm 2008; 23: 759-766.

20. Omür O, Akgün A, Ozcan Z, Sen C, Ozkiliç H. Clinical implications of diffuse hepatic uptake observed in postablative and post-therapeutic I-131 scans. Clin Nucl Med 2009; 34: 11-14.

21. Vasil'ev LI, Rozdil'skiĭ SI, Tkachenko GI. Radionuclide research on liver and kidney function in thyroid cancer after radioiodine therapy. Med Radiol (Mosk) 1987; 32: 38-41.

22. Atilgan HI, Yumusak N, Sadıc M et al. Radioprotective effect of montelukast sodium against hepatic radioiodine (131I) toxicity: A histopathological investigation in the rat model. Ankara Univ Vet Fak Derg 2015; 62: 37-43.

23. Jhummon NP, Tohooloo B, Qu S. Iodine-131 induced hepatotoxicity in previously healthy patients with Grave's disease. Thyroid Res 2013; 6: 4.

24. Shirazi A, Mihandoost E, Mahdavi SR, Mohseni M. Radio-protective role of antioxidant agents. Oncol Rev 2012; 6: e16.

25. Nair CK, Parida DK, Nomura T. Radioprotectors in radiotherapy. J Radiat Res 2001; 42: 21-37.

26. Tamura H, Takasaki A, Taketani $\mathbf{T}$ et al. Melatonin as a free radical scavenger in the ovarian follicle. Endocr J 2013; 60: 1-13.

27. Ran Y, Wang R, Gao $\mathbf{Q}$ et al. Dragon's blood and its extracts attenuate radiation-induced oxidative stress in mice. J Radiat Res 2014; 55: 699-706.

28. Esposito E, Cuzzocrea S. Antiinflammatory activity of melatonin in central nervous system. Curr Neuropharmacol 2010; 8: 228-242.

29. Kireev RA, Tresguerres AC, Garcia C, Ariznavarreta C, Vara E, Tresguerres JA. Melatonin is able to prevent the liver of old castrated female rats from oxidative and pro-inflammatory damage. J Pineal Res 2008; 45:394 402.

30. Mathes AM. Hepatoprotective actions of melatonin: possible mediation by melatonin receptors. World J Gastroenterol 2010; 16: 6087-6097.

Received October 12, 2016. Accepted November 9, 2016. 